NOTA TECNICA

\title{
COMPORTAMIENTO FENOLOGICO PRELIMINAR DE CUATRO ESPECIES FORESTALES DE AREAS INUNDABLES
}

J uan R. Baluarte Vásquez *

\section{RESUMEN}

El presente artículo reporta el comportamiento fenológico preliminar de cuatro especies forestales que ocurren en áreas inundables de la Amazonía peruana.

Debido al corto período de registro, las observaciones desarrolladas no permiten obtener conclusiones definitivas sobre el comportamiento fenológico de las especies estudiadas; sin embargo, estos resultados revelan algunas tendencias sobre las fenofases de estas especies, especialmente lo relacionado a la floración y fructificación.

\section{ABSTRACT}

Preliminary data on the phenological behavior of four tree species found in Amazonian inundate forests of Peru are reported.

The shortness of the observation period does not permit definite conclusions regarding phenological behavior of the species studied; however the results obtained reveal some tendencies as to their phenophases, particularly regarding flow ering and fruiting.

\section{INTRODUCCION}

Las altas tasas de deforestación de los bosques tropicales, exige de estrategias que permiten atenuar este problema.

Existen dos caminos para contribuir ha disminuir esta actividad, la primera mediante la utilización sustentable de los recursos forestales que incluye una serie de subestrategias y la segunda repoblando las áreas intervenidas.

* Instituto de Investigaciones de la Amazonía Peruana 
Para lograr esta segunda opción se requiere conocer los aspectos relacionados a la ecología de las especies forestales a manejar y uno de los factores fundamentales para el logro de este objetivo es el conocimiento fitofenológico.

La fitofenología es una rama de la ecología forestal que permite conocer los períodos reproductivos de las especies forestales con el propósito de programar las etapas subsecuentes como un medio para garantizar el éxito en la recuperación de las áreas intervenidas. Un buen abastecimiento de semillas y regeneración natural permitirá contar con el material reproductivo en forma oportuna para las labores silviculturales.

Desde el año 1992, se iniciaron las observaciones fenológicas de especies forestales en áreas inundables utilizadas en los modelos agroforestales de la Estación Experimental San Miguel.

Debido al excesivo número de especies seleccionadas y la escasez de recursos financieros, en el año 1993 se restringió las observaciones fenológicas a cuatro especies promisorias que mostraban tolerancia a los cambios de nivel del río. Estas especies son: Calycophyllum spruceanum "capirona", Maquira coriacea "capinurí", Ocotea aciphylla "canela moena" y Couroupita subsessilis "ayahuma".

Si bien es cierto que el número de individuos observados y el corto período registrado no permiten obtener conclusiones definitivas sobre el comportamiento fenológico de las especies estudiadas, los resultados obtenidos revelan algunas tendencias sobre las fenofases de estas especies, especialmente lo relacionado a la floración y fructificación.

Con este estudio se pretende contribuir al conocimiento de los períodos reproductivos de las especies forestales seleccionadas para el Proyecto Sistemas Integrales de Producción en A reas Inundables.

Las observaciones de campo se realizaron en los bosques de Allpahuayo y Cantagallo, ambos ubicados en las cercanías de la ciudad de Iquitos.

\section{REVISION BIBLIOGRAFICA}

La fenología es el estudio de los fenómenos biológicos acomodados a cierto ritmo periódico, como: la brotación, la florescencia, la maduración de los frutos, etc. Como es natural estos fenómenos se relacionan con el clima de la localidad en que ocurren y viceversa, de la fenología se pueden sacar consecuencias relativas al clima y, sobre todo el microclima, cuando ni uno ni otro se conocen debidamente Font Quer (1985). 
La fenología forestal es un campo de la ecología de importancia científica y tecnológica Fournier (1974) citado por Fournier y Charpantier (1975).

Permite prever la época de la reproducción de los árboles y por lo tanto determinar los períodos de recolección de semillas o material reproductivo forestal, que permitirá el abastecimiento normal de las mismas, para la producción de plantones. Asímismo, ayuda a determinar las épocas de diseminación de semillas lo que facilita el establecimiento de parcelas bajo manejo de regeneración natural. Finalmente, ayuda a comprender y conocer ciertos hábitos alimenticios relacionados con la fauna y planificar actividades turísticas ya que la floración y algunas características fenológicas le imparten belleza y colorido al paisaje en ciertas épocas del año Trucios (1986).

Las observaciones de los ciclos fenológicos de especies forestales en la zona se iniciaron a principios de la década del 70 con 2,946 árboles del Arboreto de J enaro Herrera que incluyen 267 especies determinadas Ríos (1991). Estas observaciones fueron contrastados con los datos meteorológicos con el objeto de relacionar el clima con los ritmos de reproducción de los árboles Gautier \& Spichiger (1986). Finalmente Rios (1991) procesa los datos sobre fenología de 108 especies del mismo lugar.

En un intento por superar las limitaciones que se tenía por el desconocimiento de la fenología de las especies forestales, en el Bosque Nacional Alexander von Humboldt, en 1974 se puso en marcha la recopilación y registro de todos los datos sobre la fenología que fueron colectados de varias fuentes dando posteriormente como resultado en 1977 el calendario fenológico provisional de 53 especies mostrando las épocas de floración, fructificación, maduración y diseminación Trucios (1986).

En la zona de Selva Central (Pichanaki, San Ramón, Villarica y Oxapampa), desde 1979 se iniciaron las observaciones fenológicas de especies forestales confeccionando con esos datos un calendario fenológico preliminar Trucios (1986); sin embargo, es necesario destacar que las observaciones fueron realizadas ocasionalmente, por lo que la información obtenida contribuye muy poco al conocimiento de las fenofases de las especies estudiadas.

Con respecto al número de la muestra Fournier y Charpantier (1975) sostienen que para el estudio fenológico de los árboles tropicales es recomendable el empleo de una muestra de 10 individuos por especie, seleccionada en orden de aparición en el bosque. Sin embargo,cuando esto no es posible, una muestra de cinco individuos escogidos al azar o 
en forma sistemática también es suficiente para este tipo de estudio. En lo que respecta a la frecuencia en las observaciones, es deseable que éstas se lleven a cabo quincenalmente, pero una información por mes suministra también información fenológica sobre la comunidad forestal.

\section{MATERIALES Y METODOS}

\subsection{Lugar de Ejecución}

El levantamiento de la información de campo se realizó en el bosque aledaño a la ciudad de lquitos. Los lugares específicos corresponden a los bosques de Allpahuayo y Canta Gallo.

La ciudad de lquitos se encuentra ubicada en la margen izquierda del río Amazonas, distrito de lquitos, provincia de Maynas, Región Loreto; geográficamente está sobre coordenadas 03? 45' de LS y 73운 $15^{\prime}$ de LO.

\subsection{Vías de acceso a los lugares seleccionados}

Allpahuayo está ubicada en el km. 21 de la carretera Iquitos-Nauta, para llegar hasta allí se desplaza en automóvil en un tiempo de 45 minutos por carretera desde lquitos.

Cantagallo, está localizada en la margen izquierda del río Amazonas a 30 minutos de lquitos navegando en sentido contrario al río en bote motor de $25 \mathrm{HP}$.

\subsection{Características fisiográficas del terreno donde habitan las especies forestales.}

El bosque de Allpahuayo está sobre una terraza alta de topografía ondulada con algunas quebradas serpenteadas que se desbordan cuando llueve acondicionando algunas especies a este tipo de hábitat.

Cantagallo comprende grandes áreas aluviales que se inundan con las avenidas del río Amazonas, la topografía del terreno es plano facilitando la inundación de éstas áreas, los árboles en promedio se sumergen hasta una altura aproximada de $1.50 \mathrm{~m}$ desde de la base. 


\subsection{Clima}

Los datos meteorológicos recogidos entre 1981-1990 demuestran que se trata de un clima típicamente ecuatorial.

\subsubsection{Temperatura}

La temperatura media anual es de 26.3 ㄷ; oscila a lo largo del año entre $25.5 \stackrel{\circ}{\circ}$, media del mes de julio y 26.8 oC media del mes de noviembre. Los valores extremos son de 24.4 으 (agosto 1983) y 28.2 으 (marzo de 1983).

La temperatura absoluta más baja $\left(13^{\circ} \mathrm{C}\right)$ se registró en julio 1981, conociéndose este fenómeno como "frío de San J uan". La más alta (hasta $36.8^{\circ} \mathrm{C}$ ) se puede encontrar en dos épocas febrero 1985 y agosto, setiembre y octubre de 1988.

\subsubsection{Precipitación}

Las precipitaciones varían entre $62 \mathrm{~mm}$ (enero 1985) y $565 \mathrm{~mm}$ (enero 1989).

$\mathrm{Si}$ bien es cierto que el ritmo anual no está bien marcado, al evaluar los valores medios de cada mes identificamos que la estación más seca corresponde al período junio, julio, agosto y setiembre; esta estación es bastante notoria en 1983. Además hay una estación seca menos importante pero corta, conocida con el nombre de "veranillo", se lo ubica algunas veces en los meses de diciembre a marzo: enero 1985 (62 mm), marzo 1985 (90 $\mathrm{mm}$ ) y diciembre 1989 (82 $\mathrm{mm})$.

En total la precipitación anual es en promedio de 2821.9 mm. Los años 1981, 1982 y 1987 fueron los más lluviosos (más de 3300 mm), el año 1983 el más seco (2453 mm).

\subsubsection{Nivel del río Amazonas}

El nivel del río Amazonas varía entre 105.49 m (agosto 1983) y $120.13 \mathrm{~m}$ (mayo 1982).

El período de creciente más alto corresponde a los meses de abril, mayo y junio de 1982 y el más bajo a los 
meses de julio, agosto y setiembre de 1983.

En total, el período de creciente más alto se registra en los meses de abril, mayo y junio, y el más bajo los meses de agosto y setiembre.

\subsection{METODO Y PROCEDIMIENTO EMPLEADO}

\subsubsection{Selección de las especies forestales}

De un total de 12 especies forestales instaladas en un experimento agroforestal en terraza aluvial de formación reciente, se seleccionó 4 especies en base a la tolerancia a la inundación. De acuerdo al informe de Alván, Paredes y Rojas correspondiente al año 1994, las especies forestales que soportaron la inundación que duró cerca de 90 días fueron las siguientes: Calycophyllum spruceanum (Bentham) Hooker f. ex Schumann "capirona", Maquira coriacea (Karsten) C. C. Berg "capinurí" Ocotea aciphylla (Ness) Mez "canela moena" y Couroupita subsessilis Pilger "ayahuma".

Estas especies muestran resultados alentadores para la reforestación en áreas inundables, por esta razón es necesario ampliar los estudios en aspectos como fenología, propagación y otros más.

\subsubsection{Ubicación y marcado de los árboles}

Por referencia de pobladores del lugar, se visitaron los bosques de Allpahuayo y Cantagallo, en dichos lugares se ubicaron árboles adultos de las especies seleccionadas. De inmediato se procedió a marcarlos con números de la serie natural, abriendo espacio de $10 \mathrm{~m}$ de radio alrededor de la base del árbol para facilitar la recolección de semillas y regeneración natural; adicionalmente se abrieron trochas para el ingreso hasta los árboles marcados.

Se marcaron en total 15 árboles correspondiendo seis a ayahuma, tres a capinurí, tres a capirona y tres a canela moena. La totalidad de los árboles de las tres primeras especies están ubicadas en Cantagallo, mientras que los de canela moena están ubicados en Allpahuayo. 


\subsubsection{Identificación botánica de las especies seleccionadas}

La identificación botánica de las especies seleccionadas se realizó en el Herbario Amazonense de la Universidad Nacional de la Amazonía Peruana, contando para ello con el apoyo de personal especializado. En este recinto se confrontó el material botánico con las excicatas que se encuentran codificadas en el Herbario, este primer nivel de identificación fue complementado con la revisión de la Flora del Perú de Mac Bride.

\subsubsection{Registro de Observaciones Fenológicas}

Las observaciones fenológicas se registraron en libretas de campo por un período de 3 años, estos datos fueron vaciados a formularios estructurados previamente, las fenofases observadas fueron: floración, fructificación, foliación, diseminación y defoliación; cada una de ellas se identificó mediante los códigos $F, P, A, S$ y $D$; respectivamente.

Las observaciones se realizaron inicialmente en forma mensual, posteriormente trimestral debido a las limitaciones económicas, es por esa razón que faltan datos para el caso de la especie "capinurí".

De acuerdo al código antes mencionado, los árboles numerados fueron observados y anotados en libretas de campo, así por ejemplo si el árbol 1 de capirona estaba en floración, se anotaba una $F$, si presentaba frutos una $P$ y así sucesivamente.

\subsubsection{Revisión del Registro Fenológico.}

Los datos vaciados en los formularios fueron revisados extrayendo el número de observaciones por año y por especie, procediendo luego al cálculo de los valores medios del período de floración y fructificación. 


\subsubsection{Análisis del inicio de la floración y fructificación}

Se ha hecho un análisis cuidadoso de cada una de estas fenofases para determinar el período de reproducción de las 4 especies seleccionadas, estas fenofases fueron confrontadas con las curvas climatológicas (precipitación, temperatura y nivel del río) para observar la relación entre ambos parámetros de acuerdo al método ensayado por Gautier y Spichiger (1986).

Sobre la base de la información de campo, intentaremos clasificar a las especies estudiadas en los siguientes grandes grupos:

3.5.6.1 Especies monomodales estrictos

Aquí están incluidas las especies que muestran una floración cada año, que ocurre durante la estación seca o al inicio del período lluvioso.

\subsubsection{Especies monomodales de transición}

En este grupo la floración se realiza durante la gran estación seca o después, pudiendo llegar a florecer en otras épocas del año.

\subsubsection{Especies bimodales}

Aquí se incluirán las especies que presentan dos períodos marcados de floración, tan importante uno como otro, siempre a finales de la estación seca, es decir seguido de estación lluviosa.

\subsubsection{Especies arrítmicas}

En este grupo la floración no está bien definida, es decir las especies no muestran un período privilegiado de floración con respecto a los factores climáticos. 


\section{RESULTADOS}

Con el propósito de que el estudio fenológico esté al servicio de la programación de las actividades silviculturales, hemos focalizado nuestro análisis a los períodos de floración y fructificación, llegando a obtener los siguientes resultados:

\subsection{Período de Floración}

El análisis de la duración de la floración de las 4 especies ha permitido observar que la variación de esta fenofase es de 1 a 3 meses, en promedio la floración dura 2.5 meses y está ubicada mayormente al inicio de la estación seca pudiendo extenderse en algunos casos hasta la estación lluviosa.

\subsection{Intervalo entre la floración y el inicio de la fructificación}

Es difícil observar con precisión este período transicional debido a las características de nuestros bosques; sin embargo, se ha determinado que hay una variación de 1.5 a 3.5 meses; en promedio se obtuvo 2 meses de intervalo entre estas dos fenofases.

\subsection{Período de fructificación}

El tiempo de duración de la fructificación oscila entre 1 a 5 meses, en promedio la fructificación ocurre en 3 meses. Está ubicada en la estación lluviosa mayormente.

4.4 Clasificación de las especies por grupos de acuerdo al período de floración en relación al clima.

Especie monomodal estricta: Capirona y canela moena. Especie bimodal : Ayahuma

\subsection{Calendario fenológico de floración y fructificación}

Los registros fenológicos de los árboles observados han permitido armar el siguiente calendario fenológico preliminar (Cuadro 1). 


\section{Cuadro 1}

Calendario fenológico preliminar de floración y fructificación.*

\begin{tabular}{|c|c|c|c|c|c|c|c|c|c|c|c|c|c|}
\hline \multirow[t]{2}{*}{ ESPECIE } & \multicolumn{12}{|c|}{ MESES / FENOFASES } & \multirow[t]{2}{*}{ GRUPO } \\
\hline & $E$ & $\mathrm{~F}$ & M & A & M & J & J & A & S & $\mathrm{O}$ & $\mathrm{N}$ & $\mathrm{D}$ & \\
\hline $\begin{array}{l}\text { Capirona } \\
\text { Capinuri } \\
\text { Avahuma } \\
\text { Canela } \\
\text { moena }\end{array}$ & $\mathrm{F}$ & $\begin{array}{l}F \\
P\end{array}$ & $\begin{array}{l}F \\
P\end{array}$ & $P$ & $\mathrm{~F}$ & $\mathrm{~F}$ & $\mathrm{~F}$ & $\begin{array}{l}F \\
P\end{array}$ & $\begin{array}{l}F \\
P\end{array}$ & $\begin{array}{l}F \\
P\end{array}$ & $\begin{array}{l}F \\
P\end{array}$ & $\begin{array}{l}F \\
F P\end{array}$ & $\begin{array}{l}\text { MONOMOD. } \\
\text { BIMOD. } \\
\text { MONOMOD. }\end{array}$ \\
\hline
\end{tabular}

* Período de observación: 3 años

\section{DISCUSION}

Si bien es cierto que el número de individuos observados y el corto período de observación desarrollado no permiten obtener conclusiones definitivas sobre el comportamiento fenológico de las especies estudiadas, esta entrega revela algunas tendencias sobre las fenofases de estas especies.

La escasez de recursos económicos incidieron para que las observaciones fenológicas se realizaran con frecuencias bastante largas, al principio se observó mensualmente y posteriormente en forma trimestral. Fournier y Charpantier (1975) recomiendan que en casos extremos una información mensual suministra también la fenología de la comunidad forestal.

Estas limitaciones se reflejan en vacíos de información especialmente en el caso de capinurí cuya única observación de su período reproductivo fue registrado en diciembre de 1993 anotándose la fase de floración.

Con respecto al período de floración nuestras observaciones indican que esta puede durar entre 1 a 3 meses con un promedio de 2.5 meses coincidiendo mayormente con el inicio de la estación seca pudiendo extenderse en algunas casos hasta el inicio de la estación lluviosa. La transición entre el período de floración a la fructificación es progresivo y heterogéneo no se da en forma abrupta, el promedio de esta fase es de 2 meses; la fructificación toma un tiempo de 1 a 5 meses con un promedio de 3 meses y está ubicada generalmente en la época lluviosa. En 
síntesis nuestros resultados guardan mucha aproximación con los obtenidos por Gautier \& Spichiger (1986) y con los de Ríos (1991).

La contrastación entre estas fenofases y las curvas climatológicas muestran una correspondencia entre floración de las plantas y el clima de la localidad, así la floración está estrechamente relacionada con la estación seca; este aserto coincide con las observaciones de numerosos autores en diferentes partes del mundo intertropical. Es más, no solamente se interrelaciona con la estación seca sino que también la contrastación de las fenofases con el nivel del río; para nuestro caso específico nos ha permitido observar que algunas especies adaptan su diseminación a la vaciante del río asegurando que la semilla germine en tierra firme y con regadío natural proveniente de las lluvias asegurando el éxito en el crecimiento de la regeneración natural. Es decir la interacción en la naturaleza se da de manera concatenada autorregulando los organismos dentro del ecosistema. Dentro de este sistema probablemente la fauna silvestre también juega un rol importante en la diáspora de las especies forestales, este dato no fue posible registrar debido a que no fue el propósito de este estudio.

El calendario fenológico preliminar (Cuadro 1) nos permite apreciar que "capirona" florece una sola vez al año en la estación seca clasificándose obviamente dentro del grupo monomodal; "ayahuma" florece en períodos largos iniciándose al final de la estación seca extendiéndose hasta la estación lluviosa en forma simultánea con la fase de fructificación ubicándose de esta manera dentro del grupo bimodal. La "canela moena" muestra un solo período de floración acompañado de fructificación en la estación lluviosa incluyéndose dentro del grupo monomodal; al respecto Ríos (1991) clasifica a esta última especie dentro del grupo bimodal, probablemente esta clasificación sea correcta debido a que el registro comprende un período largo de 12 años, por esta razón es necesario continuar con las observaciones por mayor número de años. 


\section{CONCLUSIONES}

- La floración de la mayoría de las especies estudiadas muestran estrecha correspondencia con la estación seca y el inicio de la estación lluviosa.

La fructificación y diseminación de las semillas de algunas especies ocurren en la época de vaciante del río y estación lluviosa asegurando de esta manera el prendimiento de la semilla en tierra firme acompañado del riego natural proveniente de las precipitaciones pluviales.

- El calendario fenológico contrastado con las curvas climatológicas nos permiten agrupar las especies en los siguientes grupos: Monomodal: capirona y canela moena; bimodal: ayahuma.

\section{BIBLIOGRAFIA}

AlVAN, J ., PAREdes, E. y ROJ AS, G. 1994. Informe final de la actividad evaluación de especies forestales de áreas inundables de San Miguel. IIAP. 8 p.

FONT QUER, P. 1985. Diccionario de Botánica. Barcelona. (España): Ed. Labor, 1244 p.

FOURNIER, L. y CHARPANTIER, C. 1975. El tamaño de la muestra y la frecuencia de las observaciones en el estudio de las características fenológicas de los árboles tropicales. Costa Rica. TURRIALBA V. 25(1): 45 - 48

GAUTIER, L. \& SPICHIGER R. 1986. Ritmos de reproducción en el estrato arbóreo del arboretum J enaro Herrera (provincia de Requena, departamento de Loreto, Perú) Contribución al Estudio de la flora y de la vegetación de la Amazonía Peruana X. Suiza. Candollea V 41 (1): 193 - 207.

RIOS, F. 1991. Estudio fenológico de 108 especies forestales del arboretum terraza alta (ATA) J enaro Herrera - Loreto Perú. Tesis para optar el título de Ingeniero Forestal. UNAP. Iquitos. (Perú). 104 p. 
TRUCIOS, T. 1986. Calendario fenológico para 55 especies forestales del Bosque Nacional Alexander von Humboldt Pucallpa, Perú. Nota técnica №2. Instituto Nacional Forestal y de Fauna (INFOR), Centro Forestal y de Fauna (CENFOR) XII - Pucallpa. 28 p. 